\title{
Rapidly growing mass in the pancreas: intraductal Candida infection in a chronic recurrent pancreatitis
}

\author{
Csaba Tóth $^{1}$, Hyon-Seok Lee ${ }^{2}$, Sebastian Heikaus ${ }^{3}$ \\ 1. University Clinic Cologne, Institute of Pathology and Biobank of Center for Integrative Oncology, Cologne, Germany. \\ 2. Evangelisches Krankenhaus Düsseldorf, Düsseldorf, Germany. 3. Zentrum für Pathologie Essen-Mitte, Essen, Germany.
}

Correspondence: Csaba Tóth. Address: University Clinic Cologne, Institute of Pathology and Biobank of Center for Integrative Oncology (ClO), Kerpener Str. 62, D-50937, Cologne, Germany. E-mail: csaba.toth@uk-koeln.de

Received: May 12, 2014

Accepted: J une 27, 2014

Online Published: July 8, 2014

DOI : $10.5430 /$ crcp.v1n2p146

URL: http://dx.doi.org/10.5430/crcp.v1n2p146

\section{Abstract}

Pancreatic fungal infection, especially Candidal infection occurs in almost every case associated with previous abdominal interventions, previous injury of the pancreas and severe acute necrotising pancreatitis. Reports on fungal infections in chronic pancreatic disease, however, are rare. Thus, we here report the extremely rare case of a 34-year-old man with chronic pancreatitis aggravated by Candidal colonisation, which clinically appeared as a rapid growing mass in the head of the pancreas two years after a complicated cholecystectomy. Below, we discuss the role of the infection in chronic pancreatitis and point out the differential diagnostic problems in this rare case.

\section{Key words}

Chronic pancreatitis, Pancreatic fungal infection, Rapidly growing pancreatic mass

\section{I ntroduction}

Pancreatic infections are usually caused by bacterial infections (E. coli etc.) and can be monomicrobial or polymicrobial. In rare cases Candida spp. can be cultured from the necrotic tissue in patients suffering from severe necrotising pancreatitis ${ }^{[1-6]}$, but fungal infections usually form only part of a polymicrobial infection and should be regarded as fungal superinfection (as detected in up to $40 \%$ of the cases) ${ }^{[3,5,7]}$. The most frequently isolated Candida spp. is Candida albicans, which is closely followed by Candida tropicalis, Candida glabrata and Candida krusei ${ }^{[1-3]}$. Candida glabrata and C. krusei occur more frequently in comorbid patients (elderly, transplanted and neutropenic patients or neonates) ${ }^{[8,9]}$. In addition, abdominal surgical intervention is a known risk factor for Candida infections ${ }^{[1]}$, and can obviously uphold and provoke symptoms years after the operation. In this context only one case has been reported so far in literature describing a case with intraluminal Candidiasis in the pancreatic ducts ${ }^{[10]}$.

The case presented here widens the spectrum of differential diagnoses of rapidly growing cystic pancreatic masses in patients with chronic pancreatitis symptoms caused by pancreatic fungal infection. 


\section{Case report}

We report the case of a 34-year-old male patient who was admitted to the hospital for further observation and elucidation of epigastric pain and rapidly growing pancreatic lesion. Two years earlier, he had undergone a complicated cholecystectomy (due to gall bladder stones with chronic cholecystitis). The procedure resulted in numerous subsequent complications such as abdominal abscess and postoperative biliary pancreatitis. Among the life style factors nicotin abuse and occasional alcohol consumption should be mentioned.

Abdominal sonography, gastroduodenoscopy and magnetic resonance cholangiopancreatography (MRCP) were performed to elucidate the cause of the epigastric pain. The first abdominal sonography showed an echo poor area of $62 \mathrm{~mm} \times 60 \mathrm{~mm}$ in the head of the pancreas and extremely dilated intra- and extrahepatic bile ducts. Three days later the control sonography already revealed an $11 \mathrm{~cm} \times 5 \mathrm{~cm}$ inhomogenic cystic, echo poor area with not only dilated but also massively congested biliary ducts. Although the image-guided diagnostic procedures (MRT, CT [see Figure 1] and sonography) favoured the diagnosis of a complicated pseudocyst of the pancreas, they could definitively not exclude a malignant process. In contrast to the morphologic findings, the labor tests showed neither inflammatory nor cholestatic changes. The tumor markers (CEA $3.6 \mathrm{ng} / \mathrm{ml}$, CA19-9 $57.1 \mathrm{U} / \mathrm{ml}$ ) and the HIV test were not pathognomic either.

Figure 1. Computer tomography of pancreatic mass, diagnosed as a complicated pseudocystic lesion, indefinite for malignancy

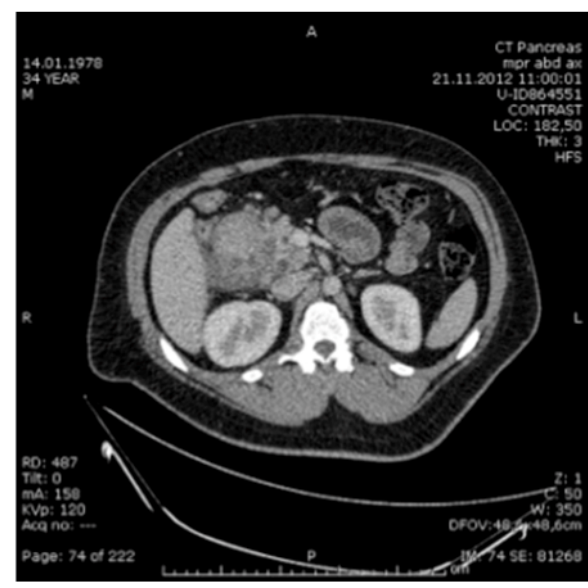

As a result, on the basis of clinical findings and the rapid progress of the disease, pylorus-sparing pancreaticoduodenectomy of Whipple was performed on the patient.

Subsequently, routine hematoxylin-eosin slides, periodic acid Schiff basis (PAS) and Grocott were also stained. Furthermore DNA extraction was performed from the formalin-embedded tissue to confirm a possible fungal or tuberculotic infection.

Macroscopically, multiple cysts communicating with the intrapancreatic ducts of up to $1.6 \mathrm{~cm}$ in diameter were detected in the head of the pancreas in an area of $3.7 \mathrm{~cm}$ in diameter. The ducts were dilated and filled with concrements. Microscopically, the dilated ducts showed erosive and ulcerative changes as well as squamous epithelial metaplasia nearby (see Figure 2). Multiple concrements with abundant fungal colonisation resembling morphologically Candida spp. were seen in the PAS and Grocott stain forming both hyphae and pseudohyphae (see Figure 3 and 4, respectively). Candida spp. was found to be Candida albicans with PCR (first pair of primers were ITS5 and ITS4 and the second pair of primers was INT1 and INT2 [MWG Biotech, Ebersberg, Germany]). Real-time PCR reactions were performed with a Roche LightCycler 2.0 Instrument (Roche Diagnostics, Germany).

Furthermore, the pancreas parenchym was in an advanced stage of pancreatic fibrosis and severe chronic recurrent pancreatitis with glandular atrophy. On the contrary, Ziel-Nielsen stain and molecular tests for mycobacterial gene sequences (MYCO-Direct 1.7, Chipron, Germany) proved to be negative. 

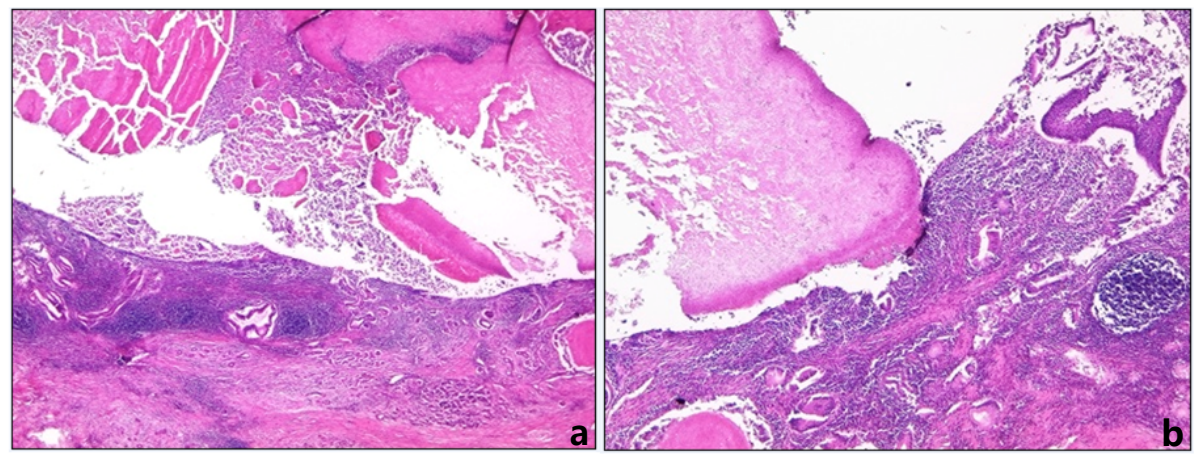

Figure 2. Hematoxylin-eosin staining showing acute and chronic inflammatory changes and the luminal concrements (a) and squamous metaplasia in the main pancreatic duct (b)

Figure 3. Periodic acid Schiff basis (PAS) stain for detection of microbial organisms. Note the abundance of mycelia and hyphae.

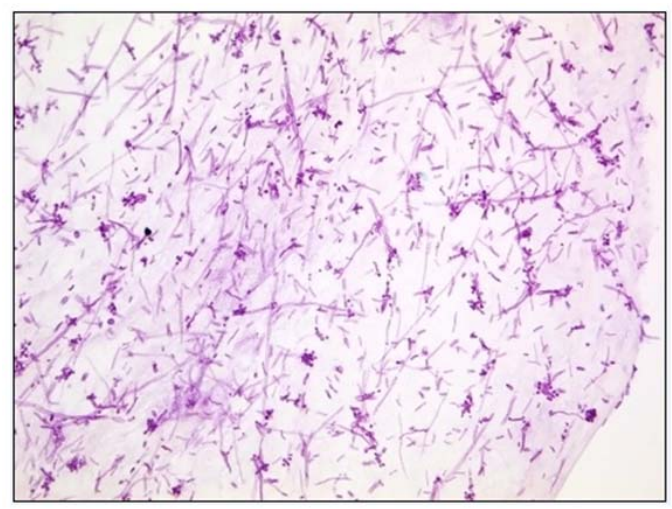

Figure 4. Grocott stain especially for fungal detection. Fungi would be later characterised with PCR analysis as Candida albicans.

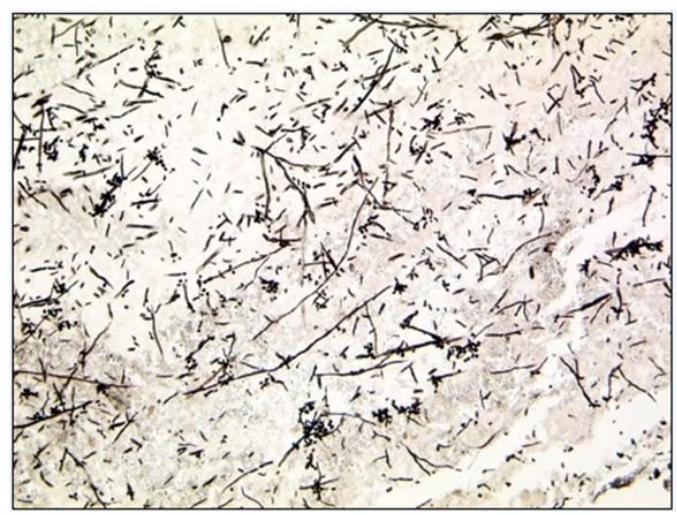

\section{Discussion}

Fungal, especially Candida infections of the pancreas are rare, but their incidence is increased in immune-suppressed patients or in those who have had surgical intervention. Chronic fibrosing pancreatitis with intraluminal concrements associated with Candida infection seems to be a rare entity and it was only reported on one occasion in 1991 in literature ${ }^{[10]}$.

Rapidly expanding pancreatic lesions are in almost every case and alarm for a tumorous process. However, next to malignant processes, some diseases of differential diagnostic importance have to be also considered such as pancreatic pseudocyst or pancreatic abscess and, as we showed, fungal colonisation of pancreatic duct stones can be associated with chronic pancreatitis. Intraluminal concrements with significant stenosis and the protein-rich pancreatic juice can cause the dilation of pancreatic and biliary ducts, whereas fungal colonisation can lead to ascending infection, building intrahepatic 
cholangitis and liver abscess. Furthermore, Candida albicans, the most significant pathogenic species, is a major cause of invasive fungal infection in the intensive care units ${ }^{[12]}$.

Most publications about pancreatic fungal infections discuss different types of lesions as being implicated in fungal infections: (1) acute necrotising pancreatitis, (2) pancreatic abscess, (3) pancreatic pseudocysts and, as in our presented case, (4) intraductal fungal infection ${ }^{[3,6,11,13]}$. Candida infection seems to appear more frequently in the acute lesions such as acute necrotising pancreatitis ${ }^{[14,15]}$. As mentioned in the introduction, the exact frequency of fungal infections in these clinical settings is still unknown, ranging up to $40 \%{ }^{[3,5,7,13]}$.

The rare occurrence of fungal infection in healthy pancreas suggests that this organ is protected from fungal infections, but pancreatic injury can damage this protective barrier. The most likely route of infection is a direct infection from the gastrointestinal tract by penetration through the normal duodenal wall (transmural seeding), but nosocomial infections by surgical intervention and abdominal drainage also appear plausible sources of fungal ingress. Furthermore, the role of biliary/duodenopancreatic reflux is also discussed ${ }^{[1,4,10,16]}$. Finally, the haematogenous spread of fungi to pancreatic tissue in immunocompromised patients is also probable ${ }^{[1-4,8]}$. However, almost all of the reported cases of Candida spp. infection are proved to have developed after a pancreas injury ${ }^{[3]}$.

Surgical intervention combined with debridement with antifungal therapy was the chosen treatment in our case. The lack of improvement in the condition of the patient with a suspicious mass of unclear dignity and rapid enlargement causing stenosis as well as abdominal discomfort were the indications for surgery. As shown in the literature, deterioration or lack of improvement in the course is an absolute indication for surgical therapy (necrosectomy, debridement) ${ }^{[1,3]}$.

In conclusion, the case presented above demonstrates that in contrast to most of the cases reported so far, pancreatic fungal infections can appear years after an abdominal intervention or pancreatic injury, such as pancreatitis, apart from acute infection and can obviously stay asymptomatic for a long time. The exact role of Candida spp. in the exacerbation of a chronic pancreatitis still remains unclear, but we believe that a change in the ductal microenvironment (progression of ductal dilation with intraductal concrements leading to stagnation of pancreatic juice) can be accounted for it.

In the case reported above, the fungal infection of the pancreas appeared as a rapid growing tumour mass with duodenal stenosis. Based on our experience, we suggest that in case of pancreatic pseudotumor/rapid growing process, the role of fungal infection should be considered. Fungi can easily be overlooked when colonising a stone. The recognition of Candida infection is vital for further clinical therapy, because the antifungal therapy after surgery is mandatory for the recovery of the patient. The treatment of local Candida infection is surgical necrosectomy and subsequent systemic antifungal therapy (with amphothericin B or fluconazole) is strongly advised as early as possible ${ }^{[3]}$.

\section{Conclusion}

Fungal infections, though being very rare, should be added to differential diagnosis in case of rapidly progressive cystic pancreatic masses in patients with chronic pancreatic disease and especially after abdominal trauma or surgical interventions.

\section{References}

[1] Kumar, S., et al. A Rare Case of Pancreatic Abscess due to Candida Tropicalis. J Glob Infect Dis. 2011; 3(4): 396-8. PMid: 22224007. http://dx.doi.org/10.4103/0974-777X.91067

[2] Chakrabarti, A., et al. Candida in acute pancreatitis. Surg Today. 2007; 37(3): 207-11. PMid: 17342358. http://dx.doi.org/10.1007/s00595-006-3371-x 
[3] Shanmugam, N., et al. Pancreatic fungal infection. Pancreas. 2003; 27(2): 133-8. PMid: 12883261. http://dx.doi.org/10.1097/00006676-200308000-00005

[4] Isenmann, R., et al. Characteristics of infection with Candida species in patients with necrotizing pancreatitis. World J Surg. 2002; 26(3): 372-6. PMid: 11865377. http://dx.doi.org/10.1007/s00268-001-0146-9

[5] Gloor, B., et al. Acute pancreatitis: threat of fungal infection. Pancreatology. 2001; 1(3): 213-6. PMid: 12120197. http://dx.doi.org/10.1159/000055813

[6] Trikudanathan, G., U. Navaneethan, S.S. Vege. Intra-abdominal fungal infections complicating acute pancreatitis: a review. Am J Gastroenterol. 2011; 106(7): 1188-92. PMid: 21731015. http://dx.doi.org/10.1038/ajg.2010.497

[7] Aloia, T., et al. Candida in pancreatic infection: a clinical experience. Am Surg. 1994; 60(10): 793-6. PMid: 7944045.

[8] Munoz, P., et al. Candida tropicalis fungaemia: incidence, risk factors and mortality in a general hospital. Clin Microbiol Infect. 2011; 17(10): 1538-45. PMid: 20718804. http://dx.doi.org/10.1111/j.1469-0691.2010.03338.x

[9] Horn, D.L., et al. Epidemiology and outcomes of candidemia in 2019 patients: data from the prospective antifungal therapy alliance registry. Clin Infect Dis. 2009; 48(12): 1695-703. PMid: 19441981. http://dx.doi.org/10.1086/599039

[10] Chung, R.T., R.H. Schapiro, A.L. Warshaw. Intraluminal pancreatic candidiasis presenting as recurrent pancreatitis. Gastroenterology. 1993; 104(5): 1532-4. PMid: 8482466.

[11] Keiser, P., S. Keay. Candidal pancreatic abscesses: report of two cases and review. Clin Infect Dis. 1992; 14(4): 884-8. PMid: 1576283. http://dx.doi.org/10.1093/clinids/14.4.884

[12] Gullo, A. Invasive fungal infections: the challenge continues. Drugs. 2009; 69 Suppl 1: 65-73. PMid: 19877737. http://dx.doi.org/10.2165/11315530-000000000-00000

[13] King, N.K., et al. Trends in fungal colonization of pancreatic necrosis in patients undergoing necrosectomy for acute pancreatitis. HPB (Oxford). 2005; 7(2): 120-3. PMid: 18333174. http://dx.doi.org/10.1080/13651820510028837

[14] Zhang, W.Z., et al. Rapid diagnosis of fungal infection in patients with acute necrotizing pancreatitis by polymerase chain reaction. Asian J Surg. 2002; 25(3): 209-13. http://dx.doi.org/10.1016/S1015-9584(09)60177-9

[15] Lee, V.T., et al. Infected pancreatic necrosis--an evaluation of the timing and technique of necrosectomy in a Southeast Asian population. Ann Acad Med Singapore. 2006; 35(8): 523-30. PMid: 17006578.

[16] Runkel, N.S., et al. The role of the gut in the development of sepsis in acute pancreatitis. J Surg Res. 1991; 51(1): 18-23. http://dx.doi.org/10.1016/0022-4804(91)90064-S 